\title{
Antipsychotic Use in Community Mental Health Service
}

\author{
Selim El-Badri, Adib Essali \\ Adult Mental Health Services, Waikato District Health Board, Hamilton, New Zealand \\ Email: Selim.El-Badri@waikatodhb.health.nz
}

How to cite this paper: El-Badri, S. and Essali, A. (2020) Antipsychotic Use in Community Mental Health Service. Open Journal of Psychiatry, 10, 45-52.

https://doi.org/10.4236/ojpsych.2020.102006

Received: February 3, 2020

Accepted: February 24, 2020

Published: February 27, 2020

Copyright $\odot 2020$ by author(s) and Scientific Research Publishing Inc. This work is licensed under the Creative Commons Attribution International License (CC BY 4.0).

http://creativecommons.org/licenses/by/4.0/

\begin{abstract}
Background: The use of antipsychotic medication remains an essential treatment modality for schizophrenia and related psychosis. However, there is a globally recognised variation in prescribing which is attributed to multiple factors. Guidelines set the expected standard and criteria for evidence based prescribing practice of antipsychotic medication. The aim of this clinical audit was to review antipsychotic prescribing patterns in adult patients with a history of schizophrenia or related psychosis attending community outpatients' clinics and to verify the uniformity of this prescribing with clinical guideline recommendations. Methods: Information about the use of antipsychotic medication was collected in a sample of a 100 patients with a history of psychosis in Waikato region to investigate the consistency of antipsychotic medication prescribing practice using standard guidelines. Identifying inconsistent patterns of prescribing will offer opportunities for intervention and advancement of best and safe practice. Results: Most patients (86\%) were maintained on a single antipsychotic medication within the recommended dose range. $27 \%$ of the patients were prescribed clozapine alone or in combination with other antipsychotic medication making clozapine the most frequently prescribed antipsychotic medication in our sample. Nearly half of the patients (48\%) were on depot antipsychotic injections of which $20 \%$ were first-generation antipsychotic injections. Conclusions: Prescribing patterns of antipsychotics amongst our group of patients seem to be consistent with current evidence-based guidelines particularly with regards to class, frequency and dose range. Further research is required to assist in the formulation of Clozapine augmentation evidence-based guidelines.
\end{abstract}

\section{Keywords}

Audit, Antipsychotics, Psychosis, Community Clinics 


\section{Introduction}

Antipsychotic medication is the mainstay of effective management of schizophrenia and related psychosis [1] [2] [3]. However, significant variations in the prescribing patterns of antipsychotic medication have been reported nationally and internationally and may have been influenced by a number of factors including clinical features (longer duration of illness, psychiatric hospital setting) and medication use (type of antipsychotic, multiple medications, decreased or increased use of typical vs atypical, concurrent use of oral and long-acting injectable antipsychotic), and personal practice. Audit and feedback have been shown to be an effective intervention in closing the gap between recommended and routine clinical practice for antipsychotic prescribing.

Information about the use of antipsychotic medication was collected in a sample of a 100 patients with a history of psychosis in Waikato region to investigate the consistency of antipsychotic medication prescribing practice using standard guidelines. Identifying inconsistent patterns of prescribing will offer opportunities for intervention and advancement of best and safe practice.

\section{Materials and Methods}

\subsection{Sample \& Tools}

A retrospective examination of the medical records of a 100 patients with diagnosis of psychotic disorder attending community mental health services was undertaken. A specially designed checklist and spreadsheet were designed and used to collect demographic details, psychiatric diagnoses and treatment. The details of prescribed antipsychotic medication were obtained using guidelines criteria as benchmark, such as use of monotherapy, initial target dose, records of poor response to monotherapy, use of multiple antipsychotic medication, and reasons for that and records of insufficient response, and strategies to manage that including dose optimisation, and cross-over switch to another antipsychotic medication or to multiple medications. Information about other relevant clinical variables that might indicate illness complexity were also collected and examined in relation to the criteria of insufficient response which included illness duration, frequency of hospitalizations and the current rating on the Global Assessment of Functioning (GAF).

\subsection{Criteria \& Standards}

The audit criteria and standards were based on the Australasian guidelines for treatment of schizophrenia and related psychosis [3] which emulate the global endorsement of good prescribing practice. It is recommended that only one antipsychotic agent at a time should be prescribed at the minimum effective dose, unless it has been clearly demonstrated that symptoms are resistant to monotherapy. Adequate sequential trials of two antipsychotic medications, of which at least one should be a second-generation antipsychotic agent, should have been conducted. If these trials are unsuccessful, and adherence is known to be good, 
then clozapine should be considered. If there has been poor or uncertain adherence, or it is the individual's preference, long-acting injectable antipsychotic medication should be considered.

\section{Results}

Demographic and diagnostic characteristics of the patients $(\mathrm{n}=100)$ are presented in Table 1. Category and percentages of antipsychotic medication use are shown in Figure 1.

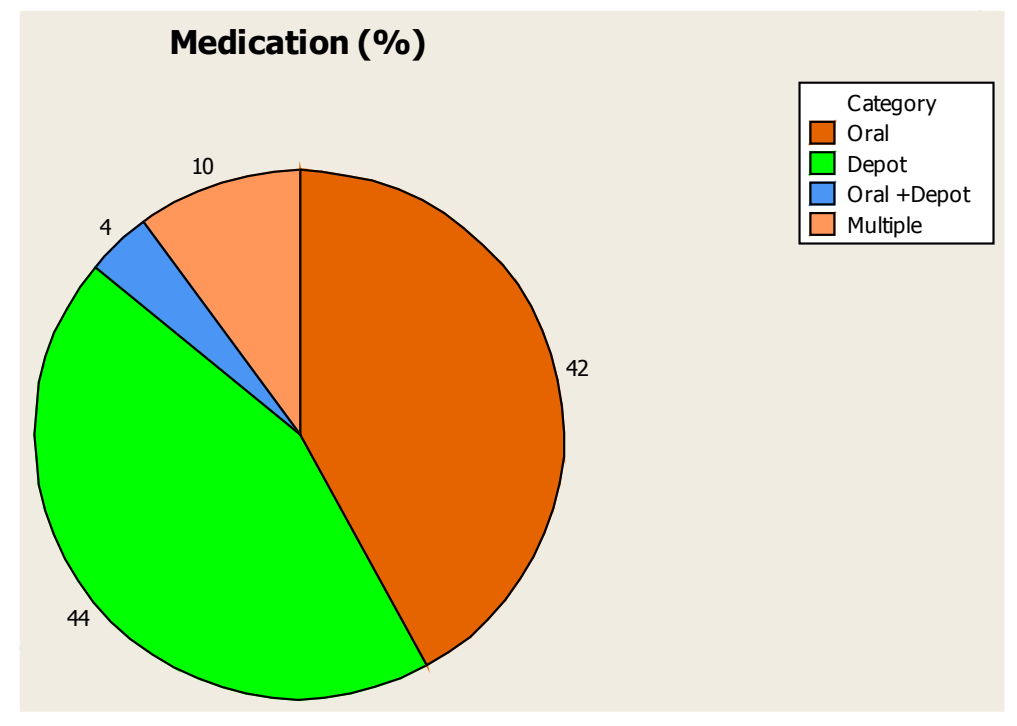

Figure 1. Category and percentages of antipsychotic medication use.

Table 1. Patient demographic and diagnostic characteristics.

\begin{tabular}{ccccc}
\hline Characteristic & $\begin{array}{c}\mathbf{n} \\
\text { (total }=100)\end{array}$ & $\begin{array}{c}\text { Duration of } \\
\text { Illness, years, } \\
\text { Mean (SD) }\end{array}$ & $\begin{array}{c}\text { Number of } \\
\text { hospitalisations } \\
\text { Mean (SD) }\end{array}$ & $\begin{array}{c}\text { GAF Mean } \\
(\text { SD })\end{array}$ \\
\hline Gender & 65 & $15.78(11.36)$ & $7.00(7.64)$ & $73.31(11.26)$ \\
Male & 35 & $15.94(12.98)$ & $5.91(7.01)$ & $73.43(10.49)$ \\
Female & & & & \\
Ethnicity & 59 & $18.9813 .16)$ & $7.83(8.09)$ & $75.08(11.31)$ \\
NZ Pakeha & 36 & $12.33(7.86)$ & $5.31(6.25)$ & $71.53(10.06)$ \\
Māori & 2 & $6.00(7.07)$ & $2.00(2.83)$ & $70(0.00)$ \\
Mixed & 3 & $2.67(0.577)$ & $1.67(1.15)$ & $63.33(11.55)$ \\
Other & & & & \\
Diagnosis & 83 & $16.20(11.81)$ & $6.87(7.12)$ & $72.59(10.77)$ \\
Schizophrenia & 10 & $18.90(13.30)$ & $8.00(10.97)$ & $74.50(10.92)$ \\
Schizoaffective & 5 & $6.80(7.63)$ & $1.60(0.89)$ & $86.00(8.94)$ \\
Delusional disorder & $5.00(9.90)$ & $2.00(1.41)$ & $67.50(3.54)$ \\
\hline Psychotic Disorder NOS & 2 & & & \\
\hline
\end{tabular}


Out of the 100 patients, 42 were on oral antipsychotic medication, 44 were on long-acting depot injections, 4 were on a combination of oral and injectable antipsychotics and 10 were on multiple antipsychotics (Figure 1). Nearly half (48) of the patients in our sample were on depot antipsychotic injections, either as a single agent or combined with oral antipsychotics. Regardless of the route of administration, most patients were on a second-generation antipsychotic (SGA) and 22 patients were on a first-generation antipsychotic (FGA). Nearly all medications were prescribed within the recommended dose range.

$27 \%$ of the patients were prescribed clozapine alone or in combination with other antipsychotic medication. Average clozapine daily dose was $363 \mathrm{mg}$ and the dose range was between $250 \mathrm{mg}$ and $700 \mathrm{mg}$. Paliperidone was prescribed for $23 \%$ of the patients, alone or in combination with other antipsychotic medication. The average dose was $100 \mathrm{mg}$ and the range was between $50 \mathrm{mg}$ and 150 mg administered four weekly.

20 patients were prescribed first-generation antipsychotic (FGA) depot injections. These preparations included flupenthixol decanoate (6 patients), zuclopenthixol decanoate (5 patients), haloperidol decanoate (6 patients) and fluphenazine decanoate ( 3 patients). Average dose and range were within recommended levels.

Olanzapine alone or in combination with other antipsychotic medication was prescribed for $16 \%$ of the patients. Most patients were on oral olanzapine (average daily dose $18.75 \mathrm{mg}$ ) and only 2 patients were on olanzapine depot injection (average dose $335 \mathrm{mg}$ ). 5 patients were on oral risperidone (average dose $5.8 \mathrm{mg}$ ) and 5 were on risperidone consta long-acting injections (average dose $47.50 \mathrm{mg}$ ). 8 patients were on quetiapine (average dose $479 \mathrm{mg}$ ) and 2 were on oral FGA drugs.

Of the 27 patients who were on clozapine, 7 were prescribed an additional antipsychotic medication. The additional antipsychotics were haloperidol (1 patient), aripiprazole ( 1 patient), quetiapine ( 2 patients), and paliperidone palmitate (3 patients).

Most atypical injectable long-acting antipsychotics were used as monotherapy. Paliperidone palmitate was used in combination with other antipsychotic medication only for 4 of the patients; in combination with clozapine for 3 patients and with risperidone for one patient. Typical (traditional) depot antipsychotics were also mostly used as monotherapy (16 out of 20 patients). The use of multiple medications was noted in 4 patients; flupenthixol decanoate was combined with oral chlorpromazine and risperidone for one patient, zuclopenthixol decanoate was combined with oral olanzapine for one patient, and haloperidol decanoate was combined with oral amisulpride for one patient and with oral olanzapine for another patient.

Generally, $86 \%$ of patients were on monotherapy and only $14 \%$ of the patients were on multiple medications. Patients on multiple medications were mostly $(93 \%)$ on the same class antipsychotic medication. In qualifying the reasons for the use of multiple medications, no reasons were reported for 3 patients $(21 \%)$ 
and there were records of insufficient response to monotherapy for 11 patients (79\%). None of these additional antipsychotics were used for comorbidities or multiple diagnoses in our sample. Initial dose optimization was reported in $7 \mathrm{pa}$ tients (58\%) of this group and switch over to another antipsychotic medication was reported in 2 patients (17\%). There were no significant differences between patients on antipsychotic monotherapy and patients on multiple antipsychotic medications in terms of duration of illness, number of hospitalizations, and current GAF scores (Figure 2).

\section{Discussion}

The findings of this audit of antipsychotic medication use for patients with psychosis in a community mental health setting were comparable with data reported previously. There were more males $(65 \%)$ in our sample than females and Māori were fairly represented comprising $36 \%$ of the sample (see Table 1).

Clozapine alone or in combination with other antipsychotic medication was the most prescribed antipsychotic and was prescribed for $27 \%$ of the patients. This is a lower than previously reported rates of clozapine use in New Zealand. It has been reported that the use of clozapine for schizophrenia in New Zealand has increased from $21.0 \%$ in 2000 to $32.8 \%$ in 2004 [4]. In the present audit, Pakeha (European New Zealanders) were prescribed clozapine more frequently than Maori (19\% Vs $8 \%$ respectively). Our audit has shown that clozapine was augmented with a variety of other antipsychotics in $7 \%$ of patients which did not seem to follow any specific imperative due to lack of evidence-based data and guidelines concerning clozapine augmentation [5].

Our finding that nearly half of patients (48\%) were on depot antipsychotic injections, either as a single agent or combined with oral antipsychotics is similar to the pattern and rates reported previously and may be related to issues with

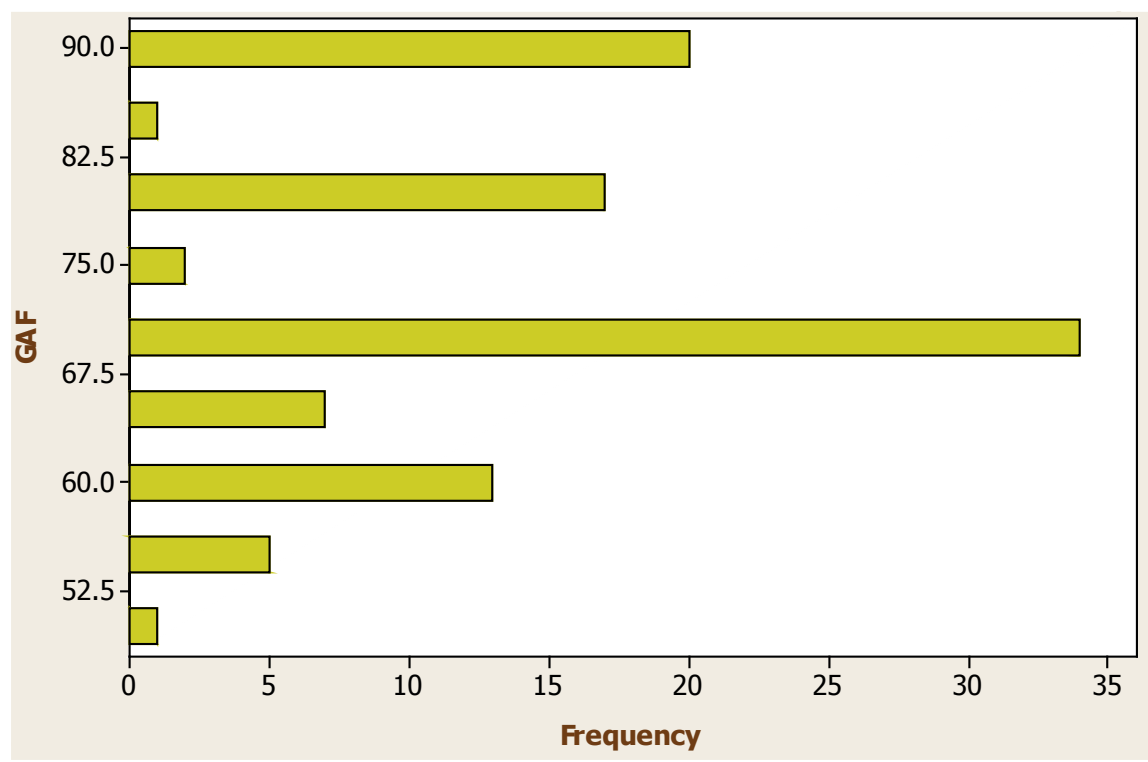

Figure 2. Patient current (GAF) scores. 
poor adherence to oral medications. This is also consistent with the literature and treatment guidelines for the management of this group of patients [6]. In addition, the second most prescribed antipsychotic was paliperidone (23\%) alone or in combination with other antipsychotic medication. Of note is the very low rate of use of olanzapine depot injections (2\%). Side effects of weight gain and the requirement of waiting for observation for a minimum of two hours after the injection are a possible explanation for the low rate of use. Some patients are not prepared to wait that long and this takes up a significant amount of nurses' time.

Typical depot antipsychotics were used for $20 \%$ of our patients occupying the 3rd place. This is an indication that these preparations still have a role to play in the treatment of patients with schizophrenia and related psychosis [6].

Olanzapine alone or in combination with other antipsychotic medication was prescribed for $16 \%$ of the patients and came 4 th. Most patients were on oral olanzapine, with the exception of 2 patients who were on olanzapine pamoate depot injections (discussed above).

The prescribing of multiple antipsychotic medications is not uncommon in routine clinical practice. It continues to be a commonly-utilized strategy to manage patients with schizophrenia and related psychotic disorders. The extent of this practice varies widely depending on geographical, regional, clinical, demographic and prescriber characteristics [2]. It also varies considerably between different regions in the same country [7]. Literature shows that use of two or more antipsychotic medications occurs in $4 \%$ to $35 \%$ of outpatients and $30 \%$ to $50 \%$ of inpatients [8]. Our finding of $14 \%$ of patients was simultaneously prescribed two or more antipsychotic medications is within the range of reported rates. In addition, most atypical injectable long-acting antipsychotics in our sample were used as monotherapy. Compared with monotherapy, a combination of antipsychotics may improve symptoms but may not make any difference in other important outcome measures such as relapse or hospitalization [9]. An association between illness complexity and consequent management difficulty and the use of multiple medications has often been reported [10]. In the present audit, the concurrent use of multiple antipsychotics was mostly considered for patients with documented evidence of insufficient response to monotherapy. Furthermore, such use was not correlated with any of the other variables including gender, ethnicity, and duration of illness or the number of past psychiatric hospitalizations. This finding is consistent with the results of similar audits from New Zealand [11] and Australia [7].

\section{Limitations}

This audit was retrospective and did not involve an evaluation of the severity of symptoms, and involuntary treatment, among patients receiving multiple medications. The audit was confined to adult outpatient community mental health setting in the Waikato region. The findings may be different for patients cared for in different settings. 


\section{Conclusion}

This audit showed that clozapine was used in our service more commonly than previously thought. More patients were prescribed atypical than typical antipsychotics regardless of the route of administration which is consistent with the current trend of prescribing. Relatively smaller proportion of patients were prescribed multiple antipsychotic medications simultaneously as a strategy to manage poor response to monotherapy with evidence of mostly good documentation of the reasons for such practice. This audit has been of value to the clinicians involved in terms of examining current practice and also establishing a baseline with which future changes can be compared and implemented.

\section{Conflicts of Interest}

The authors declare no conflicts of interest regarding the publication of this paper.

\section{References}

[1] Tandon, R. (2011) Antipsychotics in the Treatment of Schizophrenia: An Overview. Journal of Clinical Psychiatry, 72, 4-8. https://doi.org/10.4088/JCP.10075su1.01

[2] Gallego, J.A., Bonetti, J., Zhang, J., Kane, J.M. and Correll, C.U. (2012) Prevalence and Correlates of Antipsychotic Polypharmacy: A Systematic Review and Meta-Regression of Global and Regional Trends from the 1970s to 2009. Schizophrenia Research, 138, 18-28. https://doi.org/10.1016/j.schres.2012.03.018

[3] Galletly, C., Castle, D., Dark, F., et al. (2016) Royal Australian and New Zealand College of Psychiatrists Clinical Practice Guidelines for the Management of Schizophrenia and Related Disorders. Australian and New Zealand Journal of Psychiatry, 50, 410-472. https://doi.org/10.1177/0004867416641195

[4] Wheeler, A., Humberstone, V. and Robinson, G. (2006) Trends in Antipsychotic Prescribing in Schizophrenia in Auckland. Australasian Psychiatry, 14, 169-174. https://doi.org/10.1080/j.1440-1665.2006.02273.x

[5] Barber, S., Olotu, U., Corsi, M. and Cipriani, A. (2017) Clozapine Combined with Different Antipsychotic Drugs for Treatment-Resistant Schizophrenia. Cochrane Database of Systematic Reviews, 2017, Article No. CD006324. https://doi.org/10.1002/14651858.CD006324.pub3

[6] Brissos, S., Veguilla, M.R., Taylor, D. and Balanzá-Martinez, V. (2014) The Role of Long-Acting Injectable Antipsychotics in Schizophrenia: A Critical Appraisal. Therapeutic Advances in Psychopharmacology, 4, 198-219. https://doi.org/10.1177/2045125314540297

[7] John, A.P., Gee, T., Alexander, S., Ramankutty, P. and Dragovic, M. (2014) Prevalence and Nature of Antipsychotic Polypharmacy among Inpatients with Schizophrenia Spectrum Disorders at an Australian Mental Health Service. Australasian Psychiatry, 22, 546-550. https://doi.org/10.1177/1039856214546672

[8] American Psychiatric Association (2013) Five Things Physicians and Patients Should Question. Accessed 12 February 2020.

http://www.choosingwisely.org/societies/american-psychiatric-association/

[9] Ortiz-Orendain, J., et al. (2017) Antipsychotic Combinations for Schizophrenia. Cochrane Database of Systematic Reviews, Issue 6. Article No. CD009005. 
https://doi.org/10.1002/14651858.CD009005.pub2

[10] Malandain, L., Thibaut, F., Grimaldi-Bensouda, L., et al. (2018) Correlates and Predictors of Antipsychotic Drug Polypharmacy in Real-Life Settings: Results from a Nationwide Cohort Study. Schizophrenia Research, 192, 213-218.

https://doi.org/10.1016/j.schres.2017.05.015

[11] McKean, A. and Vella-Brincat, J. (2009) Audit of Antipsychotic Polypharmacy in Christchurch. Australasian Psychiatry, 17, 336-337.

https://doi.org/10.1080/10398560902839465 